Primordial prevention of high BP from younger age is necessary to prevent future CVD.

\section{P1-531 ESTIMATION OF INCIDENCE RATE OF HEPATITIS B VIRUS HORIZONTAL INFECTION IN THE JAPANESE POPULATION IN 2005}

doi:10.1136/jech.2011.142976h.19

H Tanaka, ${ }^{*}$ S Hosono. Aichi Cancer center Research Institute, Nagoya, Japan

Introduction There have been noted that not only hepatitis B virus (HBV) vertical but horizontal infection elicits a risk of persistent $\mathrm{HBV}$ infection even in healthy adulthood. There is little information on incidence rate of HBV infection in Japan.

Methods Firstly, we estimated age-specific incidence rate of HBV infection among blood donors in Osaka in 1990s from (1) the number of HBsAg sero-converting donors (57 persons) among 185554 repeat donors who donated in Osaka between 1992 and 1993, and (2) agespecific ratio for acute $\mathrm{HB}$ incident cases reported in Japan. Secondly, we modified the age-specific rates in 1990s to estimate the rates in 2000s by using age-specific coefficients which were provided under the assumption that the incidence rate is proportional to the prevalence in the same population. In the assumption, we obtained the coefficients by comparing age-specific $\mathrm{HBsAg}$ positive rates between first time blood donors in 1990s and those in 2000s. Finally, we calculated the annual number of HBV horizontal infection in Japan in 2005, using age-specific Japanese population in 2005. Sensitivity analysis was performed by changing parameters we assumed.

Results The estimated HBV horizontal infection rates among Japanese aged 15-19, 20-24, -, 60-64 were 2.4, 11.5, 18.4, 16.5, $11.9,8.4,8.8,7.3,8.8,3.9$ per 100000 P-Y, respectively in 2005. The estimated number of HBV horizontal infection in Japan was 8,409 in 2005.

Conclusion HBV horizontal infection rate was high in young adulthood in the Japanese population.

\section{P1-532 SOFT TISSUE ORAL LESIONS AND ASSOCIATED FACTORS IN YOUNG ADULTS FROM A BRAZILIAN BIRTH COHORT}

\section{doi:10.1136/jech.2011.142976h.20}

${ }^{1} \mathrm{~S}$ Tarquinio, ${ }^{*} \mathrm{~L}$ Oliveira, ${ }^{1} \mathrm{M}$ Correa, ${ }^{2} \mathrm{M}$ Peres, ${ }^{2} \mathrm{~K}$ Peres, ${ }^{2} \mathrm{D}$ Gigante, ${ }^{2} \mathrm{~B}$ Horta, ${ }^{1}$ F Demarco. ${ }^{1}$ Federal University of Pelotas, Pelotas, Rio Grande do Sul, Brazil, ${ }^{2}$ Federal University of Santa Catarina, Florianopolis, Santa Catarina, Brazil

Introduction Few population-based studies have evaluated the prevalence of soft-tissue oral lesions in young adulthood and associated factors.

Objectives To assess the prevalence and main characteristics of oral lesions, participants self-examination and their associations with social and behaviour factors in young adults.

Methods This study was carried out nested in a population-based birth cohort from Pelotas, Brazil, started in 1982. A representative sample $(n=720)$ of all 5914 births was interviewed and examined at aged 24. Data from several life course cohort waves were used. The lesions were classified according to type and location. Multivariable Poisson regression analyses were performed.

Results The prevalence of oral lesions was 23.3\% (95\% CI 20.3 to 26.6). Papules/nodules were predominant [32.0\% (25.6 to 38.9)] followed by ulcers [26.5\% (20.5 to 33.2)]. Gingiva was the most affected site $[35.0 \%$ (28.4 to 42.0$)]$ followed by lips [21.0\% (15.6 to $27.3)$ ]; $31 \%$ of the individuals reported that had never performed oral self-examination. Adjusted analyses showed that family low socio-economic status at birth [Prevalence Ratio (PR) $=1.56(95 \%$ CI: 1.08 to 2.26)], lower motheŕs participants schooling [PR 2.79
(1.34 to 5.79)], lack of oral hygiene advices by a dentist at age 15 [PR 1.64 (1.11 to 2.41)] and be a smoker at age 22 [PR 1.41 (1.07 to 1.85)] were associated with oral lesions.

Conclusions Nearly $1 / 4$ of the sample presented oral lesion. Papules/ nodules, ulcers and erosions were the most common type of alterations of the oral mucosa. The most frequent affected oral sites were gingiva and lips. Self-examination of oral cavity should be stimulated in order to improve earlier diagnosis and treatment.

\section{P1-533 TRENDS IN PREVALENCE, PREgNANCY OUTCOME, AND SURVIVAL OF CHILDREN BORN WITH SPINA BIFIDA}

doi:10.1136/jech.2011.142976h.21

${ }^{1} \mathrm{P}$ Tennant, ${ }^{*} \mathrm{M}$ Pearce, ${ }^{2} \mathrm{M}$ Bythell, ${ }^{1,2} \mathrm{~J}$ Rankin. ${ }^{1}$ Institute of Health and Society, Newcastle University, Newcastle upon Tyne, UK; ${ }^{2}$ Regional Maternity Survey Office, Newcastle upon Tyne, UK

Introduction Spina bifida is a serious congenital anomaly associated with life-long disability. This study describes trends in the prevalence and survival of spina bifida, to 10 years, using data from a longstanding congenital anomaly register in Northern England.

Methods Cases of isolated spina bifida, delivered during 1985-2003, were identified from the population-based Northern Congenital Abnormality Survey (NorCAS). Survival status was determined from local to national records. Trends in prevalence and survival were examined by the Cochran-Armitage test and by Cox regression respectively.

Results 500 cases of spina bifida were identified during the study, including 196 (39\%) live born cases. The total prevalence of spina bifida was 7.5 (95\% CI 6.9 to 8.2 ) per 10000 total births, while the live born prevalence was 3.0 (95\% CI 2.6 to 3.4) per 10000 live births. Total prevalence decreased over time from 9.6 (95\% CI 8.4 to 11.0) per 10000 total births in 1985-1990 to 5.8 (95\% CI 4.8 to 6.9) per 10000 total births in $1997-2003(p<0.001)$. The proportion of terminations of pregnancy concurrently increased, from $40.2 \%(95 \%$ CI 34.0 to 46.6 ) in $1985-1990$ to $72.4 \%$ (95\% CI 64.5 to 79.3 ) in 1997-2003 ( $\mathrm{p}<0.001) .10$ year survival among traced live born cases ( $\mathrm{n}=195)$ was $66.6 \%$ (95\% CI 59.8 to 72.6 ), increasing with time from $60.2 \%$ (95\% CI 51.1 to 68.0 ) in $1985-1990$ to $83.3 \%$ (95\% CI 64.5 to 92.7 ) in $1997-2003$ ( $\mathrm{p}=0.002$ ).

Conclusions The prevalence of spina bifida has decreased over time while survival for live born cases has significantly increased. These data are important for counselling families when a spina bifida is detected and for health service planning.

\section{P1-534 RECURRENCE OF ADVERSE PREGNANCY OUTCOMES IN WOMEN WITH PRE-GESTATIONAL DIABETES}

doi:10.1136/jech.2011.142976h.22

${ }^{1} \mathrm{P}$ W G Tennant, ${ }^{*} \mathrm{~S}$ Prapathan, ${ }^{2} \mathrm{R}$ Bilous, ${ }^{1,3} \mathrm{R}$ Bell. ${ }^{1}$ Institute of Health and Society, Newcastle University, Newcastle upon Tyne, UK; ' ${ }^{2}$ James Cook University Hospital, Newcastle upon Tyne, UK; ${ }^{3}$ Regional Maternity Survey Office, Newcastle upon Tyne, UK

Introduction Women with pre-gestational diabetes experience an increased risk of adverse pregnancy outcome, but there are limited data on recurrence of adverse outcome. This study examined recurrence of adverse outcome in a cohort of women with pregestational diabetes from Northern England.

Methods 221 women with pre-gestational diabetes and with a first and second pregnancy during 1996-2008 were identified from the Northern Diabetes in Pregnancy Survey. Rates of adverse outcome (fetal loss at any gestation, major congenital anomaly, or infant death) for each pregnancy and recurrence rates were determined 
using descriptive approaches. Predictors of adverse outcome were examined by logistic regression.

Results 69 (31\%) first pregnancies ended in adverse outcome, including 14 (6\%) with congenital anomalies, 52 (24\%) additional fetal losses, and $3(1 \%)$ additional infant deaths. 41 (19\%) second pregnancies ended in adverse outcome, significantly less than the rate among first pregnancies ( $\mathrm{p}=0.002)$, including $21(10 \%)$ with congenital anomaly, $19(9 \%)$ additional fetal losses, and $1(<1 \%)$ additional infant death. $21(10 \%)$ women experienced an adverse outcome in both pregnancies. Adverse outcome in the first pregnancy was associated with more than double the risk of an adverse outcome in the second pregnancy $[R R=2.3$ (95\% CI 1.3 to 3.9 )]. Compared to those with no history of adverse outcome, women with recurrent adverse outcomes were more likely to be from an ethnic minority background ( $\mathrm{p}=0.01)$.

Conclusion The overall risk of adverse pregnancy outcome is lower in second pregnancies than first, but history of an adverse outcome increases the risk in the second pregnancy.

\section{P1-535 RELATIONSHIP BETWEEN CHARACTERISTICS OF SOCIAL NETWORK, HEALTH-RELATED QUALITY OF LIFE AND MORTALITY PATTERNS IN OLDER AGE. KRAKOW STUDY}

doi:10.1136/jech.2011.142976h.23

B Tobiasz-Adamczyk, ${ }^{*}$ P Brzyski, A Galas, M Brzyska, M Florek. Department of Medical Sociology, Chair of Epidemiology and Preventive Medicine, Jagiellonian University Medical College, Kraków, Poland

Introduction Direct relations between social network and mortality patterns has been well documented, but less is known the relationship between size, density, boundedness of social network, social support, and psycho-social dimensions of quality of life in older age and its direct and indirect effects on all-cause mortality.

Aim The aim of the study was to assess the characteristics of social network in older stage of life in relation to health-related quality of life and its effect on mortality.

Methods The base-line study was performed in simple random sample of 552 Krakow citizen aged $65-85$ yrs old, as a part of Central European Network on Healthy Ageing. Face to face structured interviews were based on Polish validated version of Dutch questionnaire. SF20 test, HADS, GARS, SSL12-I, de Jong-Gierveld Loneliness Scale and Cantril's ladder were used. Data on mortality was ascertained by monitoring city vital records and all-caused mortality was analysed. During 8 years $31.1 \%$ of study population died. Cluster analysis was performed and Cox proportional hazard model was used.

Results Cluster analysis distinguished different clusters for age group -75 yrs and 76 yrs and over. Cox proportional models revealed significantly highest risk of death ( $\mathrm{HR}=2.97,95 \% \mathrm{CI}(1.21$ to 7.28$)$ ) in individuals aged 76 and over who were characterised by seldom contacts with children, neighbours and others, high loneliness, low social support and low general quality of life.

Conclusions Relationship between the properties of social network and health-related quality of life remains significant predictor of mortality in advanced old age.

\section{P1-536 EPIDEMIOLOGY OF PAEDIATRIC BURN INJURIES IN SHIRAZ, IRAN IN 2009}

doi:10.1136/jech.2011.142976h.24

\begin{abstract}
$1,{ }^{2} \mathrm{H}$ Tolide-ie, ${ }^{*} \mathrm{~F}$ Sahraeian, ${ }^{2} \mathrm{Z}$ Afrasiabi, ${ }^{2} \mathrm{~A}$ A Mohammadi, ${ }^{2} \mathrm{M}$ Kherad, ${ }^{2} \mathrm{~S}$ Tolide-ie. ${ }^{1}$ Gonabad University of Medical Sciences, Gonabad, Iran; ${ }^{2}$ Shiraz Burn Research Center, Shiraz University of Medical Sciences, Shiraz, Iran
\end{abstract}

Introduction Burn injuries are very important causes of morbidity and mortality among children. The aim of this study was to determine the burn related injuries in paediatric population in Shiraz, which is one of the largest cities in the Fars province in Iran.

Methods In a cross-sectional study, we investigated 691 burn victims aged 14 years and less in year 2009 admitted to Ghotbeddin hospital, the only burn specialised centre in the South of Iran.

Results These subjects represent 31.5\% (691/2194) of all burn patients treated in that year. The children's mean age was $4 \pm 3.4$ years and their burn per cent was $5.7 \% \pm 10.9 \%$. Their sex ratio $(\mathrm{M} / \mathrm{F})$ was 1.37 and $1-6$ year children were the most frequent group $(58.3 \%)$ among paediatric patients. There were no statistical significant differences in the age $(p=0.67)$ and burn per cent $(p=0.88)$ of two sexes. Only 105 cases $(15 \%)$ were hospitalised and others were treated as out-patients. Five-hundred thirty seven cases $(78 \%)$ were from urban areas and others were rural people. Boiling water $(34 \%)$, hot tea $(20 \%)$ and hot food $(6.8 \%)$ were the three leading causes of paediatric burns. Winter had the highest incidence of paediatric burns (30\%). What's more, living room (45.4\%) and kitchen (31.4\%) were the most common places of burn incidence. The mortality rate in pediatrics was $2 \%$ (14/691).

Conclusion Most of the paediatric burn injuries happened at home by hot liquids in the colder seasons of the year. It is suggested that paediatric burn prevention should be an essential element of parental education.

\section{P1-537 RETINOPATHY OF PREMATURITY AND NEONATAL RISK FACTORS: A RETROSPECTIVE COHORT STUDY}

doi:10.1136/jech.2011.142976h.25

${ }^{1} \mathrm{H}$ Hoseini, ${ }^{1} \mathrm{Z}$ Asadi, ${ }^{2,3} \mathrm{H}$ Tolide-ie, ${ }^{*} \mathrm{~S}$ Kowsarnia. ${ }^{1}$ Department of Ophthalmology, Poostchi Ophthalmic Research Center, Shiraz, Iran; ${ }^{2}$ Gonabad University of Medical Sciences, Gonabad, Iran; ${ }^{3}$ Shiraz Burn Research Center, Shiraz University of Medical Sciences, Shiraz, Iran

Introduction Recent technological advances in neonatology have increased the survival rate of very low birth weight infants, which has led to a correspondingly increased incidence of retinopathy of prematurity (ROP).

Objective To identify neonatal risk factors for severe ROP in premature babies.

Methods A retrospective cohort study was undertaken In Shiraz from January 2006 to April 2010. All premature neonates with gestational age (GA) of $<34$ weeks or birth weight (BW) of $<2000 \mathrm{~g}$ were included in the study.

Results During 5 years of study, 1095 premature infants were analysed. There were $732(66.8 \%)$ infants did not develop ROP, 363 $(33.2 \%)$ infants presented with various degrees of ROP (249 infants without treatment and 114 infants were treated with laser). We compared 2 groups of neonates, who were treated with laser and those who didn't received any treatment. Univariate logistic regression showed that GA $[\mathrm{OR}=0.68(95 \%$ CI 0.62 to $0.75)$ ], $\mathrm{BW}[\mathrm{OR}=0.997$ (95\% CI 0.996 to 0.998$)$ ], length $[\mathrm{OR}=0.84$ (95\% CI 0.80 to 0.89$)$ ], and head circumference (HC) $[\mathrm{OR}=0.73$ (95\% CI 0.66 to 0.80$)$ ] had significant inverse relationships with developing ROP and there is no statistical relationship between sex of neonates and ROP ( $p$ value $=0.054$ ). However, after adjustment in multivariate logistic model, only GA and HC remained in the model as independent risk factors. Adjusted OR for H.C was 0.8 (95\% CI 0.71 to 0.90) and Adjusted ORs for, GA $\leq 28$ weeks, and, 28 weeks $<\mathrm{GA}<32$ weeks, were $4.32(95 \%$ CI 1.76 to 10.57$)$ and 3.5 (95\% CI 1.56 to 7.83$)$ in comparison with , $\mathrm{GA} \geq 32$ weeks.

Conclusion Gestational age and head circumference at birth were the most important risk factors for severe ROP in our study. 